\title{
Cerebral Microbleeds, CSF p-Tau, and Cognitive Decline: Significance of Anatomic Distribution
}

\author{
G.C. Chiang, J.C. Cruz Hernandez, K. Kantarci, C.R. Jack Jr, and M.W. Weiner; for the Alzheimer's Disease Neuroimaging Initiative
} O- ${ }^{\mathrm{EBM}} \mathrm{M}$

\begin{abstract}
BACKGROUND AND PURPOSE: Cerebral microbleeds are associated with aging, hypertension, and Alzheimer disease. Microbleeds in a lobar distribution are believed to reflect underlying amyloid angiopathy, whereas microbleeds in the deep gray matter and infratentorial brain are commonly seen with hypertension. However, it is unknown how microbleeds in either distribution are related to Alzheimer pathogenesis. The purpose of this analysis was to test whether lobar and deep gray/infratentorial microbleeds demonstrate differential associations with CSF amyloid- $\beta$ and phosphorylated tau 181 protein levels and longitudinal cognitive decline.
\end{abstract}

MATERIALS AND METHODS: A total of 626 subjects (151 cognitively normal, 389 with mild cognitive impairment, and 86 with Alzheimer disease) from the Alzheimer's Disease Neuroimaging Initiative who had undergone 3T MR imaging and lumbar puncture were included in the analysis. The number and location of microbleeds were assessed visually. Associations between lobar or deep gray/infratentorial microbleeds with CSF amyloid- $\beta$ levels, abnormal CSF phosphorylated tau 181 protein levels, and longitudinal cognitive decline were assessed by using ordinary least-squares, logistic, and mixed-effects regression models while adjusting for covariates.

RESULTS: Having $\geq 3$ lobar microbleeds was associated with lower levels of CSF amyloid- $\beta(P=.001)$. After adjusting for CSF amyloid- $\beta$ level, lobar microbleeds were independently associated with a higher likelihood of having an abnormal CSF phosphorylated tau 181 protein level $(P=.004)$. Lobar microbleeds were associated with accelerated longitudinal cognitive decline $(P=.007)$. Deep gray/infratentorial microbleeds revealed no significant associations.

CONCLUSIONS: The distribution of microbleeds revealed different associations with amyloid- $\beta$ and phosphorylated tau 181 protein levels and cognition. Lobar and deep gray/infratentorial microbleeds should be considered separately with regard to Alzheimer disease pathogenesis.

ABBREVIATIONS: $\mathrm{A} \beta=$ amyloid $-\beta ; \mathrm{AD}=$ Alzheimer disease; $\mathrm{ADAS}=$ Alzheimer Disease Assessment Scale; $\mathrm{ADNI}=$ Alzheimer's Disease Neuroimaging Initiative; $A P O E=$ apolipoprotein $E ;$ p-tau $=$ phosphorylated tau 181 protein

C erebral microbleeds, typically associated with aging, hypertension, and Alzheimer disease (AD), are common findings on gradient recalled-echo and susceptibility-weighted MR imaging sequences. In a population-based study in which a gradient recalled-echo sequence at $1.5 \mathrm{~T}$ was used, approximately $36 \%$ of

Received January 9, 2015; accepted after revision February 22.

From the Department of Radiology (G.C.C.), Division of Neuroradiology, Weill Cornell Medical College, New York-Presbyterian Hospital, New York, New York; Department of Biomedical Engineering (J.C.C.H.), Cornell University, Ithaca, New York; Department of Radiology (K.K., C.R.J.), Mayo Clinic, Rochester, Minnesota; and Department of Radiology and Biomedical Imaging (M.W.W.), University of California, San Francisco, California.

This work was supported in part by National Institutes of Health National Center for Advancing Translational Sciences/Clinical and Translational Science Center grant UL1 TR000457-06.

Paper previously presented at: Annual Meeting of the American Society of Neuroradiology, May 20-23, 2013; San Diego, California.

Please address correspondence to Gloria C. Chiang, MD, Department of Radiology, Division of Neuroradiology, Weill Cornell Medical College, New York-Presbyterian the people $>80$ years old were found to have microbleeds, compared with only $7 \%$ of the people who were $45-50$ years old. ${ }^{1}$ In a systematic review of studies that used echo-planar and gradient recalled-echo sequences at $0.5-3 \mathrm{~T}$, hypertensive individuals were reported to be 4 times more likely than those in the general population to have microbleeds, ${ }^{2}$ particularly in association with other signs of small-vessel disease, such as white matter hyperintensities and lacunar infarcts. ${ }^{3,4}$ In a review of multiple studies that used gradient recalled-echo and SWI sequences at $1.5 \mathrm{~T}$ and 3T, people with mild cognitive impairment and Alzheimer disease were reported to have microbleeds at a prevalence of $20 \%-43 \%$ and $18 \%-32 \%$, respectively, compared with $0 \%-19 \%$ in cogni-

Hospital, 525 East 68th St, Starr Pavilion, Box 141, New York, NY 10065; e-mail: gcc9004@med.cornell.edu

- Indicates open access to non-subscribers at www.ajnr.org

Evidence-Based Medicine Level 2.

http://dx.doi.org/10.3174/ajnr.A4351 
tively normal individuals. ${ }^{5}$ In the setting of $\mathrm{AD}$, microbleeds are associated with global brain amyloidosis, seen with increased uptake on positron-emission tomography scans by using $\left[{ }^{18} \mathrm{~F}\right]$ florbetapir $^{6}$ and decreased levels of CSF amyloid- $\beta(\mathrm{A} \beta) .^{5}$

The anatomic distribution of microbleeds is believed to reflect their underlying pathology. Microbleeds located in the deep gray matter and infratentorial brain are typically seen in hypertensive individuals $\mathrm{s}^{2,7}$ and correspond to foci of hemosiderin leakage from small abnormal blood vessels. ${ }^{8}$ Conversely, microbleeds in the setting of aging and $\mathrm{AD}$ are typically lobar, at the corticosubcortical junction, ${ }^{2,7}$ and correspond to amyloid- $\beta$ deposition along vessel walls, ${ }^{9}$ also known as amyloid angiopathy. However, it remains unclear whether microbleeds, either from hypertension or amyloid angiopathy, relate to tau pathology or cognitive changes that lead to Alzheimer pathogenesis ${ }^{10}$ independent of global brain amyloidosis.

The purpose of our analysis was to determine whether the distribution of microbleeds, either lobar or deep gray/infratentorial, has differential associations with downstream events in Alzheimer pathogenesis. Specifically, by using data from the multicenter Alzheimer's Disease Neuroimaging Initiative (ADNI) (adni.loni.usc.edu), ${ }^{11}$ we tested the hypotheses that lobar microbleeds 1) are associated with brain amyloidosis, reflected by lower CSF amyloid- $\beta$ levels, 2) predict the presence of tau pathology after overall brain amyloidosis is adjusted for, and 3) predict greater longitudinal cognitive decline.

\section{MATERIALS AND METHODS}

\section{Subjects}

The subjects of these analyses were 626 ADNI participants who had undergone 3T MR imaging and lumbar puncture for CSF analysis (151 cognitively normal, 389 with mild cognitive impairment, and 86 with $\mathrm{AD}$ ). The ADNI is a longitudinal multicenter observational cohort study designed to identify imaging and biochemical biomarkers for the diagnosis and monitoring of $\mathrm{AD} .^{12}$ The study was approved by the institutional review board of each of the participating institutions. Informed written consent was obtained from each participant at each site. Each of the subjects who enrolled in the ADNI-2 or ADNI-Grand Opportunity underwent a $\mathrm{T}^{*}$ gradient recalled-echo sequence, which was used to enumerate the microbleeds in the brain. Subjects were between the ages of 55 and 90 years and showed no clinical or structural evidence of a significant neurologic or psychiatric disease and no systemic medical illness or laboratory abnormalities that would interfere with follow-up. To reduce confounding by comorbid vascular disease, a subject was enrolled in the ADNI only if he or she had a low modified Hachinski score of $\leq 4 .{ }^{13}$ Cognitive function was assessed by using the Alzheimer Disease Assessment Scale (ADAS), ${ }^{14}$ which is the most widely used measure for clinical trials.

\section{MR Image Acquisition}

Each subject underwent a standardized 3T MR imaging protocol, which included $\mathrm{T} 2{ }^{*}$ gradient recalled-echo and T1-weighted 3D MPRAGE sequences (http://adni.loni.usc.edu/methods/documents/ mri-protocols/). This standardized protocol was distributed digitally to all ADNI-certified scanners and included the following parameters for the gradient recalled-echo sequence: TE, $20 \mathrm{~ms}$; TR, $650 \mathrm{~ms}$; flip angle, $20^{\circ}$; section thickness, $4 \mathrm{~mm}$; section gap, 0 $\mathrm{mm}$. Before any subject was scanned using this protocol, an ADNI phantom was used to assess linear and nonlinear spatial distortion, signal-to-noise ratio, and image contrast, which were reviewed by a single quality-control center to ensure harmonization among the sites. ${ }^{15}$

Microbleeds were quantified visually by a board-certified neuroradiologist with subspecialty certification (G.C.C.). Microbleeds were defined as hypointense lesions within the brain parenchyma that measured $<10 \mathrm{~mm}$ on the gradient recalled-echo sequence. Only microbleeds that were considered definite were included in the analysis.

Microbleeds were classified visually by location as 1) deep gray matter/infratentorial, if they involved the basal ganglia, thalami, brain stem, or cerebellum, or 2) lobar, if they involved other regions of the brain parenchyma.

\section{CSF Biomarkers}

Each subject included in this analysis underwent lumbar puncture to obtain CSF samples for quantifying levels of CSF amyloid- $\beta$ and phosphorylated tau 181 protein (p-tau). ${ }^{16}$ Briefly, each lumbar puncture was performed with a 20- or 24-gauge spinal needle at the baseline visit after an overnight fast. The CSF samples were transferred into polypropylene transfer tubes, frozen on dry ice within 1 hour after collection, and shipped on dry ice overnight to a single designated laboratory. After thawing for 1 hour at room temperature and gentle mixing, $0.5-\mathrm{mL}$ aliquots were prepared from these samples. The aliquots were then stored in bar-codelabeled polypropylene vials at $-80^{\circ} \mathrm{C}$ and measured by using the xMAP Luminex platform (Luminex, Austin, Texas) with INNO-BIA AlzBio3 (Innogenetics, Ghent, Belgium) immunoassay kit-based reagents. Monoclonal antibodies specific for $A \beta$ and $p$-tau, which have been found to be useful in predicting $\mathrm{AD}$, were used as reagents. ${ }^{17}$ Total CSF tau level was not included in this analysis, because it was not available for some of the participants. In addition, p-tau has a higher specificity and negative predictive value for ruling out the presence of $\mathrm{AD}$ with $90 \%$ probability. ${ }^{18}$

\section{Apolipoprotein E Genotyping}

Each participant underwent apolipoprotein E (APOE) genotyping at the baseline visit. Approximately $6 \mathrm{~mL}$ of blood was obtained from each participant in an ethylenediamine tetraacetic acid tube, gently mixed by inversion, and shipped at ambient temperature to a single designated laboratory within 24 hours of collection for genotyping analysis.

\section{Statistical Analysis}

All statistical analyses were programmed in STATA version 13 (StataCorp, College Station, Texas). Comparisons of baseline variables among the groups were performed by using the Wilcoxon rank sum, Fisher exact, Kruskal Wallis, or $\chi^{2}$ test, depending on the number of groups and type and distribution of the variables.

To test the hypothesis that lobar microbleeds are associated with overall brain amyloidosis, we used ordinary least-squares regression with age, sex, years of education, history of hyperten- 


\begin{tabular}{|c|c|c|c|c|c|c|}
\hline \multirow[b]{2}{*}{ Characteristic } & \multirow[b]{2}{*}{$\begin{array}{l}\text { Microbleeds } \\
\text { Absent }\end{array}$} & \multirow[b]{2}{*}{$\begin{array}{c}P \\
\text { Value }^{\mathrm{a}}\end{array}$} & \multicolumn{3}{|c|}{ Microbleeds Present } & \multirow[b]{2}{*}{$\begin{array}{c}P \\
\text { Value }^{\mathrm{b}}\end{array}$} \\
\hline & & & $\begin{array}{c}\text { Lobar Microbleeds } \\
\text { Only }\end{array}$ & $\begin{array}{l}\text { Mixed Lobar and Deep Gray/ } \\
\text { Infratentorial Microbleeds }\end{array}$ & $\begin{array}{l}\text { Deep Gray/Infratentorial } \\
\text { Microbleeds Only }\end{array}$ & \\
\hline No. of subjects & 407 & & 163 & 29 & 27 & \\
\hline Mean age (SD), y & $72(7.6)$ & $<.001^{\mathrm{c}}$ & $74(7.0)$ & $77(6.4)$ & $77(9.0)$ & .06 \\
\hline Sex, male/female & 218:189 & .15 & $100: 63$ & $17: 12$ & 14:13 & .64 \\
\hline Mean education (SD), y & $16(2.6)$ & .24 & $16(2.9)$ & $16(2.9)$ & $16(2.3)$ & .78 \\
\hline$\%$ with HTN & 47 & .28 & 46 & 72 & 67 & $.009^{d}$ \\
\hline$\%$ with APOE4 & 43 & .15 & 53 & 52 & 26 & $.03^{d}$ \\
\hline$\%$ with APOE2 allele & 7 & $>.99$ & 6 & 7 & 19 & .06 \\
\hline$\%$ with NL & 26 & .14 & 20 & 10 & 33 & .10 \\
\hline$\%$ with $\mathrm{MCl}$ & 61 & .80 & 61 & 79 & 56 & .33 \\
\hline$\%$ with $A D$ & 12 & .18 & 18 & 10 & 11 & .41 \\
\hline
\end{tabular}

Note:-HTN indicates hypertension; MCl, mild cognitive impairment; NL, normal cognitive function; SD, standard deviation.

a $P$ values compare baseline variables between those without microbleeds and those with microbleeds (combining the 3 groups with different types of microbleeds).

${ }^{\mathrm{b}} \mathrm{P}$ values compare baseline variables among the 3 groups with microbleeds.

c Significance according to the Wilcoxon rank sum test.

d Significance according to the $\chi^{2}$ test.

sion, APOE2 and APOE4 status, and diagnostic group (normal cognitive function, mild cognitive impairment, or AD) as covariates. CSF A $\beta$ values were log transformed for normality and included as the outcome variable. Lobar microbleeds were dichotomized as 0 or 1 (indicating presence or absence, respectively) and used as the predictor. The number of lobar microbleeds was then categorized as an ordinal variable of $0,1,2,3$, or $>3$ to determine whether increasing numbers of lobar microbleeds were associated with greater brain amyloidosis, reflected by decreased CSF A $\beta$ levels. The regressions were then repeated by using deep gray/ infratentorial microbleeds, categorized as dichotomous or ordinal variables, to differentiate between microbleeds that may have been associated with hypertension and those associated with amyloid angiopathy.

To determine whether lobar microbleeds are associated with tau pathology, we used logistic regression, adjusting for age, sex, years of education, history of hypertension, APOE2 and APOE4 status, and diagnostic group. CSF A $\beta$ level was also included as a covariate, dichotomized as normal or abnormal on the basis of the previously published cutoff of $192 \mathrm{pg} / \mathrm{mL},{ }^{19}$ to determine the effect of microbleeds independent of global brain amyloidosis. We then dichotomized abnormal CSF p-tau levels by using a cutoff of $23 \mathrm{pg} / \mathrm{mL},{ }^{19}$ which was used as the outcome variable. Lobar versus deep gray/infratentorial microbleeds were again included as the predictor, either as a dichotomous or ordinal variable. The sensitivity, specificity, positive predictive value, and negative predictive value of lobar microbleeds in predicting an abnormal CSF p-tau level were also calculated post hoc.

To determine whether lobar microbleeds are associated with a longitudinal change in cognition, we used the following linear mixed-effects model: $\mathrm{ADAS}_{i j}=\left(B_{0}+\beta_{0}\right)+\beta_{1} \mathrm{MCH}_{i}+\left(\beta_{2}+\beta_{3}\right.$ $\left.\mathrm{MCH}_{i}\right) t_{i j}+$ covariates $+\varepsilon_{i j}$. ADAS $i j$ represents the ADAS score of subject $i$ at time point $j, \mathrm{MCH}_{i}$ represents the presence or absence of lobar microbleeds in each subject, and $t_{i j}$ represents the time interval between ADAS tests. $B_{0}$ and $\beta_{0}$ are the coefficients for the random and fixed variations in baseline ADAS scores, respectively. The coefficient $\beta_{1}$ represents the fixed effect of having lobar microbleeds at baseline. Finally, $\beta_{2}+\beta_{3}$ are the coefficients for time-dependent changes in ADAS scores, irrespective or respec- tive, of the presence of microbleeds. The error term $\varepsilon_{i j}$ represents random noise.

\section{RESULTS}

Subject characteristics are presented in Table 1. Of the 626 subjects, 407 (65\%) had no microbleeds, whereas 219 (35\%) had at least one microbleed. Of the 219 subjects with microbleeds, 192 (88\%) had at least one lobar microbleed, and 27 (12\%) had only deep gray/infratentorial microbleeds. As expected, the subjects with microbleeds were older $(P<.001) .{ }^{1}$ Those with lobar microbleeds, either alone or in combination with deep gray/infratentorial microbleeds, were more likely to be APOE4 carriers $(P=.03)$, which has also been described previously. ${ }^{1}$ Finally, those with mixed lobar and infratentorial or only infratentorial microbleeds were more likely to have a history of hypertension $(P=.009)$.

\section{Having $\geq 3$ Lobar Microbleeds Was Associated with Abnormal Levels of CSF A $\beta$, Whereas Having Deep Gray/ Infratentorial Microbleeds Was Not}

After adjusting for covariates, having at least one lobar microbleed was associated with greater brain amyloidosis, reflected by a lower CSF A $\beta$ level, though this association did not reach statistical significance (coefficient $=-0.04 ; P=.08$ ). However, accounting for increasing numbers of microbleeds, having 3 (coefficient $=-0.30 ; P=.001)$ or $>3$ (coefficient $=-0.18 ; P=.001)$ lobar microbleeds was also associated with lower levels of CSF A $\beta$ (Table 2). Deep gray/infratentorial microbleeds were not associated with CSF A $\beta$ levels $(P=.64)$, and increasing numbers of deep gray/infratentorial microbleeds also were not associated with CSF $\mathrm{A} \beta$ levels $(P=.29-.97)$.

\section{Lobar Microbleeds Are Associated with a Higher Likelihood of Having an Abnormal CSF p-Tau Level, Independent of the CSF A $\beta$ Level; Deep Gray/Infratentorial Microbleeds Are Not Associated with Abnormal CSF p-Tau Levels}

Using logistic regression after adjusting for the CSF A $\beta$ level, having at least one lobar microbleed was associated with more than 
Table 2: Regression model demonstrating association between lobar microbleeds and CSF amyloid- $\beta$ level (log transformed)

\begin{tabular}{lcc}
\hline \multicolumn{1}{c}{ CSF A $\boldsymbol{\beta}$} & Coefficient $(\mathbf{9 5 \%} \mathrm{Cl})$ & $\boldsymbol{P}$ Value \\
\hline No. of lobar microbleeds & & \\
1 & $-0.005(-0.057$ to 0.047$)$ & .85 \\
2 & $-0.030(-0.12$ to 0.056$)$ & .49 \\
3 & $-0.30(-0.48$ to -0.12$)$ & .001 \\
$>3$ & $-0.18(-0.29$ to -0.068$)$ & .002 \\
Age & $-0.007(-0.0098$ to -0.0041$)$ & $<.001$ \\
Male sex & $-0.013(-0.055$ to -0.030$)$ & .57 \\
Years of education & $0.003(-0.0046$ to 0.011$)$ & .40 \\
APOE4 & $-0.23(-0.28$ to -0.19$)$ & $<.001$ \\
APOE2 & $0.059(-0.022$ to 0.14$)$ & .15 \\
MCI & $-0.078(-0.13$ to -0.028$)$ & .002 \\
AD & $-0.26(-0.33$ to -0.19$)$ & $<.001$ \\
History of hypertension & $-0.011(-0.052$ to 0.031$)$ & .61 \\
\hline
\end{tabular}

Note:- $\mathrm{MCl}$ indicates mild cognitive impairment.

Table 3: Regression model demonstrating association between lobar microbleeds and likelihood of an abnormal CSF p-tau level

\begin{tabular}{lcc}
\hline \multicolumn{1}{c}{$\begin{array}{c}\text { Abnormal CSF p-Tau } \\
\text { Level }\end{array}$} & $\begin{array}{c}\text { Odds Ratio } \\
(95 \% \mathrm{CI})\end{array}$ & P Value \\
\hline $\begin{array}{l}\text { No. of lobar microbleeds } \\
1\end{array}$ & $2.82(1.52-5.24)$ & .001 \\
2 & $1.24(0.51-3.01)$ & .64 \\
3 & $0.37(0.081-1.73)$ & .21 \\
$>3$ & $3.22(0.40-25.9)$ & .27 \\
Abnormal CSF A $\beta$ level & $3.40(2.16-5.34)$ & $<.001$ \\
Age & $1.01(0.99-1.04)$ & .32 \\
Male sex & $0.93(0.61-1.42)$ & .74 \\
Years of education & $0.98(0.90-1.06)$ & .56 \\
APOE4 & $2.33(1.42-3.84)$ & .001 \\
APOE2 & $1.02(0.52-2.02)$ & .95 \\
MCl & $1.05(0.67-1.67)$ & .83 \\
AD & $3.5(1.15-10.7)$ & .03 \\
History of hypertension & $0.98(0.65-1.48)$ & .92 \\
\hline
\end{tabular}

Note:- $\mathrm{MCl}$ indicates mild cognitive impairment.

double the odds of having an abnormal CSF p-tau level $(P=$ .004). The sensitivity and specificity of detecting an abnormal CSF p-tau level with the presence of at least one lobar microbleed were $35 \%$ and $82 \%$, respectively. The positive and negative predictive values of at least one lobar microbleed for an abnormal CSF p-tau level were $86 \%$ and $29 \%$, respectively.

Having deep gray/infratentorial microbleeds was not associated with abnormal CSF p-tau levels $(P=.97)$. Unlike with CSF $\mathrm{A} \beta$ levels, no dose-response relationship was seen; having one lobar microbleed was most associated with an abnormal CSF p-tau level, with an odds ratio of $2.8(P=.001)$ (Table 3$)$.

\section{Lobar Microbleeds Are Associated with Accelerated Longitudinal Cognitive Decline}

Using a linear mixed-effects model and adjusting for covariates, including CSF $A \beta$ level and diagnostic group, having at least one lobar microbleed was significantly associated with an accelerated longitudinal change in ADAS score $(P=.007)$ of 1.4 points per year compared with 0.8 points per year for those without lobar microbleeds. Furthermore, having $>3$ lobar microbleeds was significantly associated with an increase of 2.3 points per year $(P<$ .001) (Table 4). Deep gray/infratentorial microbleeds were not associated with change in ADAS scores $(P=.31)$.
Table 4: Mixed-effects regression model demonstrating an association between lobar microbleeds and longitudinal change in ADAS

\begin{tabular}{ccc}
\hline $\begin{array}{c}\text { No. of Lobar } \\
\text { Microbleeds }\end{array}$ & $\begin{array}{c}\text { Annual Change in } \\
\text { ADAS }(95 \% \mathrm{Cl})\end{array}$ & $\boldsymbol{P}$ Value \\
\hline 0 & $0.78(0.52$ to 1.03$)$ & $<.001$ \\
1 & $1.35(0.92$ to 1.78$)$ & .025 \\
2 & $0.36(-0.61$ to 1.32$)$ & .41 \\
3 & $0.50(-2.07$ to 3.07$)$ & .83 \\
$>3$ & $2.34(1.51$ to 3.18$)$ & $<.001$
\end{tabular}

\section{DISCUSSION}

The following are the major findings of our analysis: 1 ) having $\geq 3$ lobar microbleeds is associated with global brain amyloidosis, whereas having deep gray/infratentorial microbleeds is not; 2) lobar microbleeds, unlike deep gray/infratentorial microbleeds, are associated with elevated CSF p-tau levels but with no doseresponse relationship; and 3) lobar microbleeds, unlike deep gray/ infratentorial microbleeds, are associated with accelerated longitudinal cognitive decline. Overall, the results of our analysis suggest a differential association of lobar versus deep gray/infratentorial microbleeds with Alzheimer pathogenesis, which reveals the importance of lobar microbleeds in prognostication, independent of CSF A $\beta$ levels.

The first major finding, that lobar microbleeds are associated with greater overall brain amyloidosis, is concordant with the results of previous studies that showed lower CSF A $\beta$ levels ${ }^{3,20,21}$ and higher uptake on PET amyloid scans $s^{5,6}$ in people with microbleeds. Previous studies have also suggested that lobar microbleeds are more suggestive of underlying amyloid angiopathy, ${ }^{2,7,9}$ which is seen concomitantly in $78 \%-98 \%$ of postmortem examinations of the brains of people with Alzheimer disease. ${ }^{22}$ The finding that only higher numbers of lobar microbleeds were found to be associated with abnormal CSF A $\beta$ levels suggests that severe amyloid angiopathy is related more to overall brain amyloidosis and may contribute to the disease process. On the other hand, deep gray/infratentorial microbleeds, which are associated more typically with hypertension, were not associated with CSF A $\beta$ levels. Previous work also demonstrated that deep gray, not lobar, microbleeds are associated with small-vessel disease. ${ }^{23}$ Therefore, hypertension may produce deep gray/infratentorial microbleeds and small-vessel changes but is likely involved in Alzheimer pathogenesis from a nonamyloid pathway, such as decreased cognitive reserve.

The second major finding is that having at least one lobar microbleed, unlike having deep gray/infratentorial microbleeds, was associated with greater odds of having an abnormal CSF p-tau level, after adjusting for CSF A $\beta$ levels. Furthermore, in post hoc analyses, we found that the presence of at least one lobar microbleed had a high positive predictive value for an abnormal CSF p-tau level. However, the absence of lobar microbleeds had a low negative predictive value for excluding the presence of an abnormal CSF p-tau level. A recent study in a memory clinic population found a similar association between microbleeds and CSF total tau, but not CSF p-tau, levels in individuals without dementia. ${ }^{21}$ The fact that they did not find an association between microbleeds and p-tau levels may reflect technical differences in the assay or the fact that $\mathrm{p}$-tau is more specific for neurodegeneration in 
cohorts with more comorbid vascular disease. Nonetheless, both of our studies revealed an association between microbleeds and elevated levels of forms of CSF tau. One hypothesis is that microbleeds might reflect damaged microvasculature, resulting in decreased blood flow to neurons, ischemia, neuronal degeneration, and increased tau pathology. In postmortem studies, p-tau seems to have increased aggregation around arteries and arterioles with $\mathrm{A} \beta$ in the vessel walls. ${ }^{24}$ Alternatively, microbleeds might induce enough inflammation, without duration, to cause an elevated CSF p-tau level. In an animal model, microhemorrhages were seen to trigger inflammation and activated microglia, macrophages, and lymphocytes ${ }^{9,25}$ rather than cell death or ischemia. This inflammation may be sufficient to produce neuronal release of tau into the extracellular space. This inflammation may also contribute to further Alzheimer pathogenesis. Finally, the presence of lobar microbleeds, reflecting amyloid angiopathy, may suggest that a patient is farther along in the Alzheimer disease cascade. ${ }^{10}$ In this case, lobar microbleeds, in addition to an abnormal CSF A $\beta$ level, may signal more severe underlying disease.

The third major finding is that having at least one lobar microbleed is associated with accelerated longitudinal cognitive decline. Results discussed in the literature evaluating the association between microbleeds and cognitive decline have been variable, depending on the cohort studied (community-based populations, ${ }^{26-29}$ people with a history of stroke or suspected stroke, ${ }^{30-33}$ or subjects in a memory clinic who had mild cognitive impairment/AD ${ }^{3,34-38}$ ) and whether cognition was being evaluated on a cross-sectional $^{26,30-32,34,35}$ or longitudinal ${ }^{27,33,36-39}$ basis. Most of these studies demonstrated an association between microbleeds and either global or executive impairment ${ }^{3,26,28,30-32}$ cross-sectionally, as well as increased progression of memory impairment, ${ }^{38}$ greater longitudinal changes in Mini-Mental State Examination scores, ${ }^{36}$ higher likelihood of mild cognitive impairment converting to $\mathrm{AD},{ }^{27}$ and increased risk of developing incident dementia. ${ }^{39}$ One study also found cognitive improvement after stroke if no microbleeds were present, ${ }^{33}$ which suggests a detrimental role of microbleeds on cognitive recovery. However, none of these studies adjusted for concomitant Alzheimer pathology, which may have driven the longitudinal changes in cognition. In our study, the association between lobar microbleeds and accelerated cognitive decline persisted after adjusting for CSF A $\beta$ levels, which suggests that microbleeds alone, perhaps reflecting underlying amyloid angiopathy, lead to cognitive impairment. This finding mirrors that of a postmortem study that found that moderate-to-severe amyloid angiopathy is associated with perceptual speed and episodic memory, even after adjusting for concomitant AD pathology. ${ }^{40}$ We also found that having $\geq 3$ lobar microbleeds was more associated with decreasing cognition, similar to the results of previous studies that found greater cognitive decline with $\geq 2^{39}$ or $\geq 5^{28}$ lobar microbleeds. The finding that deep/infratentorial microbleeds were not associated with cognitive decline is concordant with results of a previous study ${ }^{32}$ but differs from those of another study that found mixed, not strictly lobar, microbleeds were associated with cognitive decline. ${ }^{39}$ Again, the differential effect on cognitive decline secondary to microbleed location may hint at differing etiologies of microbleeds.
Our study has several limitations. First, the ADNI is not a community sample. The cohort consisted of more white people, people who were more highly educated, and people who had fewer comorbidities than a community population at this age. ${ }^{11}$ Furthermore, subjects were excluded from the ADNI if they had significant comorbid vascular disease, reflected by a modified Hachinski score of $>4 .{ }^{13}$ Therefore, although we did not find a significant effect on hypertension-related deep gray/infratentorial microbleeds on tau pathology and cognition, a significant association may be found in people with more significant vascular disease. As a result, generalization of these findings should be approached with caution, and further validation in prospective population-based cohorts, particularly those with concomitant vascular disease, is required.

\section{CONCLUSIONS}

The distribution of microbleeds provides clinically significant information, not only in suggesting differences in underlying etiology (ie, amyloid angiopathy versus hypertension) but also in demonstrating different associations with downstream events in Alzheimer pathogenesis (ie, tau and cognitive decline). Those who perform neuroimaging evaluations of older individuals who are being assessed for cognitive decline should consider microbleed distribution in their reports.

\section{ACKNOWLEDGMENTS}

Data used in the preparation of this article were obtained from the Alzheimer's Disease Neuroimaging Initiative database (adni. loni.usc.edu). As such, the investigators within the ADNI contributed to the design and implementation of the ADNI and/or provided data but did not participate in the analysis or writing of this report. A complete listing of ADNI investigators can be found at http://adni.loni.usc.edu/wp-content/uploads/how_to_apply/ ADNI_Acknowledgement_List.pdf.

The ADNI was launched in 2003 by the National Institute on Aging, the National Institute of Biomedical Imaging and Bioengineering, the Food and Drug Administration, private pharmaceutical companies, and nonprofit organizations as a \$60-million 5 -year public-private partnership. The primary goal of the ADNI has been to test whether serial MR imaging, positron-emission tomography, other biologic markers, and clinical and neuropsychological assessment can be combined to measure the progression of mild cognitive impairment and early Alzheimer disease. Determination of sensitive and specific markers of very early AD progression is intended to aid researchers and clinicians to develop new treatments and monitor their effectiveness and to lessen the time and cost of clinical trials.

The principal investigator of this initiative is Michael W. Weiner, MD, VA Medical Center and University of California, San Francisco. The ADNI is the result of efforts of many coinvestigators from a broad range of academic institutions and private corporations, and subjects have been recruited from $>50$ sites across the United States and Canada. The initial goal of the ADNI was to recruit 800 subjects, but the ADNI was followed by ADNIGrand Opportunity and ADNI-2. To date, these 3 protocols have recruited $>1500$ adults to participate in the research, consisting of cognitively normal older individuals, people with early or late 
mild cognitive impairment, and people with early AD. The follow-up duration of each group is specified in the protocols for ADNI-1, ADNI-2, and ADNI-Grand Opportunity. Subjects originally recruited for ADNI-1 and ADNI-Grand Opportunity had the option to be followed in ADNI-2. For up-to-date information, please see www.adni-info.org.

Data collection and sharing for this project were funded by the Alzheimer's Disease Neuroimaging Initiative (National Institutes of Health grant U01 AG024904) and Department of Defense ADNI (Department of Defense award W81XWH-12-2-0012). The ADNI is funded by the National Institute on Aging and the National Institute of Biomedical Imaging and Bioengineering and through generous contributions from the following: Alzheimer's Association; Alzheimer's Drug Discovery Foundation; Araclon Biotech; BioClinica; Biogen Idec; Bristol-Myers Squibb; Eisai; Elan Pharmaceuticals; Eli Lilly; EuroImmun; F. Hoffmann-La Roche and its affiliated company Genentech; Fujirebio; GE Healthcare; IXICO; Janssen Alzheimer Immunotherapy Research \& Development; Johnson \& Johnson Pharmaceutical Research \& Development; Medpace; Merck \& Co; Meso Scale Diagnostics; NeuroRx Research; Neurotrack Technologies; Novartis Pharmaceuticals Corporation; Pfizer; Piramal Imaging; Servier; Synarc; and Takeda Pharmaceutical Company. The Canadian Institutes of Health Research is providing funds to support ADNI clinical sites in Canada. Private sector contributions are facilitated by the Foundation for the National Institutes of Health (www.fnih.org). The grantee organization is the Northern California Institute for Research and Education, and the study is coordinated by the Alzheimer's Disease Cooperative Study at the University of California, San Diego. ADNI data are disseminated by the Laboratory for Neuro Imaging at the University of Southern California.

Disclosures: Gloria C. Chiang—RELATED: Grant: National Center for Advancing Translational Sciences/Clinical and Translational Science Center. Kejal KantarciRELATED: Grant: National Institutes of Health, ${ }^{*}$ Comments: R01AG040042, P50 AG44170/Project 2, and Minnesota Partnership for Biotechnology and Medical Genomics (PO03590201); OTHER: serves on the data safety monitoring board for Pfizer, Janssen Alzheimer Immunotherapy, and Takeda Global Research \& Development Center and receives research support from the National Institutes of Health (grant R01AG040042 [principal investigator], Mayo Clinic Alzheimer's Disease Research Center/Project one grant P50 AG16574/P1 [principal investigator], grant P50 AG44170/Project 2 [principal investigator], and grant R01 AG11378 [coinvestigator]). Clifford Jack-RELATED: Grant: National Institutes of Health (AG11378 and AG041851)*; UNRELATED: Consultancy: Eli Lily; OTHER: provides consulting services for Siemens Healthcare and receives research funding from the National Institutes of Health (grants R01-AG011378, R01-AG041851, R01-AG037551, U01-HL096917, U01AG032438, and U01-AG024904) and the Alexander Family Alzheimer's Disease Research Professorship of the Mayo Foundation. Michael Wiener-RELATED: Grant: National Institutes of Health/National Institute on Aging/National Institute of Mental Health, ${ }^{*}$ Department of Defense, ${ }^{*}$ Alzheimer's Association, ${ }^{*}$ Alzheimer's Drug Discovery Foundation, ${ }^{*}$ Merck, ${ }^{*}$ Avid, ${ }^{*}$ and the Veterans Administration*; Consulting Fee or Honorarium: Synarc, Pfizer, Janssen, KLJ Associates, Easton Associates, Harvard University, University of California, Los Angeles, Alzheimer's Drug Discovery Foundation, Neurotrope Bioscience, Avid Radiopharmaceuticals, Clearview Healthcare Partners, Perceptive Informatics, Smartfish AS, Decision Resources, Araclon, Merck, Defined Health, and Genentech; Support for Travel to Meetings for the Study or Other Purposes: Pfizer, Paul Sabatier University, MCI Group France, Travel eDreams, Neuroscience School of Advanced Studies, Danone Trading, BV, CTAD Ant Congres, Kenes International, ADRC, University of California, Los Angeles, University of California, San Diego, Sanofi-Aventis Groupe, University Center Hospital, Toulouse, Araclon, AC Immune, Nutricia, Eli Lilly, New York Academy of Sciences, National Brain Research Center, India for Johns Hopkins Medicine, Consortium for Multiple Sclerosis Centers, Northwestern University, Fidelity Biosciences Research Initiative, and University of Pennsylvania. He served on the Editorial Boards for Alzheimer's \& Dementia and MRI. He received honoraria from Pfizer, Tohoku University, and Danone Trading, BV; OTHER: has been on scientific advisory boards for Pfizer and BOLT International; has been a consultant for Pfizer, Janssen, KLJ Associates, Easton Associates, Harvard University, inThought, INC Research, University of California, Los Angeles, Alzheimer's Drug Discovery Foundation, and Sanofi-Aventis Groupe; has received funding for travel from Pfizer, Alzheimer's disease PD meeting, Paul Sabatier University, Novartis, Tohoku University, MCl Group, France, Travel eDreams, Neuroscience School of Advanced Studies, Danone Trading, BV, and CTAD ANT Congress; serves as an associate editor of Alzheimer's \& Dementia; has received honoraria from Pfizer, Tohoku University, and Danone Trading, BV; has research support from Merck, Avid, Department of Defense, and Veterans Administration; and has stock options in Synarc and Elan. *Money paid to the institution.

\section{REFERENCES}

1. Poels MM, Vernooij MW, Ikram MA, et al. Prevalence and risk factors of cerebral microbleeds: an update of the Rotterdam Scan Study. Stroke 2010;41:S103-06 CrossRef Medline

2. Cordonnier C, Al-Shahi Salman R, Wardlaw J. Spontaneous brain microbleeds: systematic review, subgroup analyses and standards for study design and reporting. Brain 2007;130:1988-2003 CrossRef Medline

3. Goos JD, Kester MI, Barkhof F, et al. Patients with Alzheimer disease with multiple microbleeds: relation with cerebrospinal fluid biomarkers and cognition. Stroke 2009;40:3455-60 CrossRef Medline

4. Wardlaw JM, Lewis SC, Keir SL, et al. Cerebral microbleeds are associated with lacunar stroke defined clinically and radiologically, independently of white matter lesions. Stroke 2006;37:2633-36 CrossRef Medline

5. Yates PA, Villemagne VL, Ellis KA, et al. Cerebral microbleeds: a review of clinical, genetic, and neuroimaging associations. Front Neurol 2014;4:205 CrossRef Medline

6. Kantarci K, Gunter JL, Tosakulwong N, et al; Alzheimer's Disease Neuroimaging Initiative. Focal hemosiderin deposits and $\boldsymbol{\beta}$-amyloid load in the ADNI cohort. Alzheimers Dement 2013;9:S116-23 CrossRef Medline

7. Park JH, Seo SW, Kim C, et al. Pathogenesis of cerebral microbleeds: in vivo imaging of amyloid and subcortical ischemic small vessel disease in 226 individuals with cognitive impairment. Ann Neurol 2013;73:584-93 CrossRef Medline

8. Fazekas F, Kleinert R, Roob G, et al. Histopathologic analysis of foci of signal loss on gradient-echo $\mathrm{T} 2^{\star}$-weighted MR images in patients with spontaneous intracerebral hemorrhage: evidence of microangiopathy-related microbleeds. AJNR Am J Neuroradiol 1999;20: 637-42 Medline

9. Schrag M, McAuley G, Pomakian J, et al. Correlation of hypointensities in susceptibility-weighted images to tissue histology in dementia patients with cerebral amyloid angiopathy: a postmortem MRI study. Acta Neuropathol 2010;119:291-302 CrossRef Medline

10. Jack CR Jr, Knopman DS, Jagust WJ, et al. Tracking pathophysiological processes in Alzheimer's disease: an updated hypothetical model of dynamic biomarkers. Lancet Neurol 2013;12:207-16 CrossRef Medline

11. Petersen RC, Aisen PS, Beckett LA, et al. Alzheimer's Disease Neuroimaging Initiative (ADNI): clinical characterization. Neurology 2010;74:201-09 CrossRef Medline

12. Mueller SG, Weiner MW, Thal LJ, et al. Ways toward an early diagnosis in Alzheimer's disease: the Alzheimer's Disease Neuroimaging Initiative (ADNI). Alzheimers Dement 2005;1:55-66 CrossRef Medline

13. Rosen WG, Terry RD, Fuld PA, et al. Pathological verification of ischemic score in differentiation of dementias. Ann Neurol 1980;7: 486-88 CrossRef Medline

14. Rosen WG, Mohs RC, Davis KL. A new rating scale for Alzheimer's disease. Am J Psychiatry 1984;141:1356-64 CrossRef Medline

15. Gunter JL, Bernstein MA, Borowski BJ, et al. Measurement of MRI scanner performance with the ADNI phantom. Med Phys 2009;36: 2193-205 CrossRef Medline

16. Shaw LM. PENN biomarker core of the Alzheimer's Disease Neuroimaging Initiative. Neurosignals 2008;16:19-23 CrossRef Medline

17. Hansson O, Zetterberg H, Buchhave P, et al. Association between 
CSF biomarkers and incipient Alzheimer's disease in patients with mild cognitive impairment: a follow-up study. Lancet Neurol 2006; 5:228-34 CrossRef Medline

18. Mitchell A, Brindle N. CSF phosphorylated tau-does it constitute an accurate biological test for Alzheimer's disease? Int J Geriatr Psychiatry 2003;18:407-11 CrossRef Medline

19. Shaw LM, Vanderstichele H, Knapik-Czajka M, et al. Cerebrospinal fluid biomarker signature in Alzheimer's disease neuroimaging initiative subjects. Ann Neurol 2009;65:403-13 CrossRef Medline

20. Yates PA, Sirisriro R, Villemagne VL, et al; AIBL Research Group. Cerebral microhemorrhage and brain $\boldsymbol{\beta}$-amyloid in aging and Alzheimer disease. Neurology 2011;77:48-54 CrossRef Medline

21. Kester MI, Goos JD, Teunissen CE, et al. Associations between cerebral small-vessel disease and Alzheimer disease pathology as measured by cerebrospinal fluid biomarkers. JAMA Neurol 2014;71: 855-62 CrossRef Medline

22. Kalaria RN, Ballard C. Overlap between pathology of Alzheimer disease and vascular dementia. Alzheimer Dis Assoc Disord 1999;13: S115-23 CrossRef Medline

23. Vernooij MW, van der Lugt A, Ikram MA. Prevalence and risk factors of cerebral microbleeds: the Rotterdam Scan Study. Neurology 2008;70:1208-14 CrossRef Medline

24. Williams S, Chalmers K, Wilcock GK, et al. Relationship of neurofibrillary pathology to cerebral amyloid angiopathy in Alzheimer's disease. Neuropathol Appl Neurobiol 2005;31:414-21 CrossRef Medline

25. Rosidi NL, Zhou J, Pattanaik S, et al. Cortical microhemorrhages cause local inflammation but do not trigger widespread dendrite degeneration. PLoS One 2011;6:e26612 CrossRef Medline

26. Takashima $\mathrm{Y}$, Mori $\mathrm{T}$, Hashimoto $\mathrm{M}$, et al. Clinical correlating factors and cognitive function in community-dwelling healthy subjects with cerebral microbleeds. J Stroke Cerebrovasc 2011;20:105-10 CrossRef Medline

27. Kirsch W, McAuley G, Holshouser B, et al. Serial susceptibility weighted MRI measures brain iron and microbleeds in dementia. J Alzheimers Dis 2009;17:599-609 CrossRef Medline

28. Poels MM, Ikram MA, van der Lugt A, et al. Cerebral microbleeds are associated with worse cognitive function: the Rotterdam Scan Study. Neurology 2012;78:326-33 CrossRef Medline

29. Qiu C, Cotch MF, Sigurdsson S, et al. Cerebral microbleeds, retinop- athy, and dementia: the AGES-Reykjavik Study. Neurology 2010;75: 2221-28 CrossRef Medline

30. Werring DJ, Frazer DW, Coward LJ, et al. Cognitive dysfunction in patients with cerebral microbleeds on $\mathrm{T} 2{ }^{\star}$-weighted gradient-echo MRI. Brain 2004;127:2265-75 CrossRef Medline

31. Patel B, Lawrence AJ, Chung AW, et al. Cerebral microbleeds and cognition in patients with symptomatic small vessel disease. Stroke 2013;44:356-61 CrossRef Medline

32. Gregoire SM, Scheffler G, Jäger HR, et al. Strictly lobar microbleeds are associated with executive impairment in patients with ischemic stroke or transient ischemic attack. Stroke 2013;44: 1267-72 CrossRef Medline

33. Tang WK, Chen YK, Lu JY, et al. Absence of cerebral microbleeds predicts reversion of vascular "cognitive impairment no dementia" in stroke. Int J Stroke 2011;6:498-505 CrossRef Medline

34. Pettersen JA, Sathiyamoorthy G, Gao FQ, et al. Microbleed topography, leukoaraiosis, and cognition in probable Alzheimer disease from the Sunnybrook dementia study. Arch Neurol 2008;65:790-95 CrossRef Medline

35. Nakata Y, Shiga K, Yoshikawa K, et al. Subclinical brain hemorrhages in Alzheimer's disease: evaluation by magnetic resonance T2*-weighted images. Ann N Y Acad Sci 2002;977:169-72 CrossRef Medline

36. van der Vlies AE, Goos JD, Barkhof F, et al. Microbleeds do not affect rate of cognitive decline in Alzheimer disease. Neurology 2012;79: 763-69 CrossRef Medline

37. Haller S, Bartsch A, Nguyen D, et al. Cerebral microhemorrhage and iron deposition in mild cognitive impairment: susceptibilityweighted MR imaging assessment. Radiology 2010;257:764-73 CrossRef Medline

38. Ayaz M, Boikov AS, Haacke EM, et al. Imaging cerebral microbleeds using susceptibility weighted imaging: one step toward detecting vascular dementia. J Magn Reson Imaging 2010;31:142-48 CrossRef Medline

39. Miwa K, Tanaka M, Okazaki S, et al. Multiple or mixed cerebral microbleeds and dementia in patients with vascular risk factors. Neurology 2014;83:646-53 CrossRef Medline

40. Arvanitakis Z, Leurgans SE, Wang Z, et al. Cerebral amyloid angiopathy pathology and cognitive domains in older persons. Ann Neurol 2011;69:320-27 CrossRef Medline 